\title{
Variational approach to interreflection in color images
}

\author{
Mark S. Drew and Brian V. Funt \\ School of Computing Science, Simon Fraser University, Vancouver, British Columbia V5A 1S6, Canada
}

\begin{abstract}
Received February 25, 1991; revised manuscript received February 17, 1992; accepted February 18, 1992.
Interreflections affect the colors of surfaces as they appear in images. The light reflected by one surface that then impinges upon a second surface changes the color of the overall illumination that it receives and hence the color of the light that it reflects. Both the relative colors and positions of the two surfaces affect the result. We analyze the physics of the interreflection process and extract constraints on the possible surface reflectances, ambient illumination, and geometric configuration of the surfaces. By using the calculus of variations, a finite-dimensional model of reflectance, and a one-bounce model of interreflection, we express these constraints as a set of equations that are then solved for the surface spectral reflectance functions of the surfaces, the spectrum of the ambient illumination, and local interreflection factors related to the scene geometry. The interreflection factors express how the image is altered by interreflection effects and can be used to produce an image shaded as it would appear had there been no interreflection; the surface reflectance functions provide color constancy. Although it is more complex than some previous analyses of interreflection, the variational approach is more general and relaxes some restrictive assumptions concerning the type of illumination and the number of surfaces.
\end{abstract}

\section{INTRODUCTION}

Interreflection of light is important in the generation of intensities and colors in images. For example, a white ceiling contributes a good deal to the overall lighting in a room because of the reflection of light from it. Also, because of interreflection the color of one surface in a scene can affect the apparent colors of other surfaces.

Although study of interreflection has a long history in illumination and thermal engineering, ${ }^{1-3}$ it has only relatively recently come under consideration in computer vision. One reason is that the problem is inherently nonlinear in that the mutual illumination impinging upon a surface 1 from another surface 2 is in part the result of reflection from surface 2 to surface 1 . The seminal research of Koenderink and van Doorn ${ }^{4}$ formalizes this nonlinear problem for known shapes and its integral equation solution.

References 5-7 are concerned with the broad problem of recovering the mutual illumination from an image of a set of surfaces all of which can interreflect and each of which can have varying albedo. The emphasis in these papers is on solving for the albedo values under general conditions including those situations in which a surface can interreflect with itself or surfaces can partially occlude each other. Nayar et $a .^{7}$ found that shape-fromintensity schemes can succeed in spite of interreflection effects by iteratively estimating the shape and then estimating the interreflection, based on the current shape estimate, until both estimates converge.

In computer graphics the radiosity method addresses the interreflection problem more from an engineering perspective in which the emphasis (see, e.g., Ref. 2) is on finding how large patches (the walls of a room, say) interreflect, where each patch is assumed to have uniform reflectance. Generally, radiosity methods replace a single albedo by three color bands ${ }^{8,9}$ representing surface reflectance. From the underlying theory, however, it is most accurate to carry out diffuse interreflection calculations by using a whole-spectrum description of surface reflectance. In Refs. 10 and 11 we calculated the color signal spectrum reflected from a simple geometry illuminated isotropically, with patches participating in full nonlinear mutual illumination.

The goal of the variational method described in this paper is to overcome-albeit at the expense of increased complexity - some of the restrictions and limitations of our two previous approaches to the analysis of interreflection. The method in Ref. 10 obtains color constancy and shading information in the form of recovered finite-dimensionalmodel approximations of the surface reflectances and incident illumination along with interreflection factors summarizing information on the orientations and shapes of the surfaces. The method is restricted to presegmented images of only two interreflecting Lambertian surfaces under diffuse illumination. It also assumes that a point can be found on each of the surfaces at which the interreflection effects are negligible, and it relies on a one-bounce model of interreflection (i.e., multiple bounces from surface to surface are ignored). A one-bounce assumption only leads to serious problems in highly concave surface folds, regions that cause mutual illumination to dominate the direct illumination.

A different method in Ref. 12 factors an image into two parts, one with no mutual illumination and the other consisting of the mutual illumination alone. In this method surface reflectances are not determined; however, the algorithm is much simpler, and the diffuse-illumination restriction is eliminated.

In this paper the variational approach enables us to obtain the surface reflectance and interreflection factor information while dispensing with the need for the diffuse-illumination restriction, a two-patch limit, and locations with negligible interreflection. Nonetheless, we still assume a presegmented image and Lambertian reflectances and continue to rely on a one-bounce model of 
interreflection. The variational approach allows us to express all the constraints on the reflectances, the geometry, and the illumination that are all intertwined in the effects of mutual illumination.

The calculus of variations provides a method for solving an optimization problem for all the relevant parameters simultaneously. The one-bounce model of mutual illumination renders solvable the resulting equations derived by the calculus of variations, because under the onebounce assumption these equations turn into a set of nonlinear equations that can be solved iteratively by intermediate linear equations.

The variational approach serves as a useful guide for arriving at a set of equations to solve. ${ }^{13}$ Since images are sampled discretely, the same set of equations could be developed by minimizing a matrix-vector summation. We find, however, that writing the minimization in terms of continuous quantities is a useful shorthand and results in a simpler format for the resulting equations. It also provides the option of applying smoothness conditions in a straightforward manner. Such conditions were found unnecessary in simulations, even when noise was included. The advantage of not insisting on smoothness is that surfaces may be rough.

Once the shading and interreflection fields are obtained, one has in essence a shading field for each of two separate illuminations. Therefore it may be feasible to use the analysis of interreflection to carry out a type of photometric stereo method for the retrieval of surface orientation.

In Section 2 we outline the finite-dimensional linear models for surface reflectance and illumination on which our method rests. In Section 3 we introduce the variational description of the problem and address the issue of how much interreflection information can be gleaned from an image. In Section 4 we detail the resulting equations for a simplified calculation involving only two convex patches and no shading. Section 5 tests the method by simulating mutual illumination edges composed of planar sides for various materials. The usefulness of this simple geometry is that all properties can be completely determined analytically, so that recovered results can be compared with exact values. We find that the method recovers surface spectral reflectance and the illumination spectrum with reasonable accuracy and recovers a good approximation of the interreflection factor, which encapsulates surface geometry information.

\section{FINITE-DIMENSIONAL MODELS}

Natural surface spectral reflectance functions and illumination spectra have been found to be well modeled by finite-dimensional linear models. ${ }^{14-16}$ By using a set of basis spectral functions $E_{a}, a=1, \ldots, m$, we approximate illumination $E(\lambda)$ by a sum

$$
E(\lambda) \simeq \sum_{a=1}^{m} \epsilon_{a} E_{a}(\lambda)
$$

Let us restrict our attention to Lambertian surfaces. Then we can model surface spectral reflectance $S(\lambda)$ by a similar sum with another basis $S_{\mu}, \mu=1, \ldots, n$ :

$$
S(\lambda) \simeq \sum_{\mu=1}^{n} \sigma_{\mu} S_{\mu}(\lambda) .
$$

When light is reflected from a diffuse surface, the resulting spectrum, the color signal $C(\lambda)$, is formed by the product

$$
C(\lambda)=E(\lambda) S(\lambda) .
$$

Now consider the result of applying a set of filters with response functions $R_{k}(\lambda), k=1, \ldots, s$, to the color signal. For a color camera, $s=3$. If we call the resulting set of camera or eye responses $\rho_{k}$ and substitute relations (1) and (2) into Eq. (3), we have

$$
\rho_{k}=\int C(\lambda) R_{k}(\lambda) \mathrm{d} \lambda=\sum_{a=1}^{m} \sum_{\mu=1}^{n} \epsilon_{a} \sigma_{\mu} g_{a \mu k},
$$

where $^{14}$

$$
g_{a \mu k} \equiv \int E_{a}(\lambda) S_{\mu}(\lambda) R_{k}(\lambda) \mathrm{d} \lambda
$$

and indices $a, b, c, \ldots$ for illumination go from 1 to $m$; Greek indices for surface reflectance go from 1 to $n$; and index $k$ for sensor values goes from 1 to $s$.

For the case of interreflected light, the initial color signal reflects off the same or another surface, so we will need a matrix similar to $g_{a \mu k}$ but of dimension four. Let

$$
h_{a \mu \nu k} \equiv \int E_{a}(\lambda) S_{\mu}(\lambda) S_{\nu}(\lambda) R_{k}(\lambda) \mathrm{d} \lambda
$$

As an illumination basis set we choose Judd's principalcomponent analysis of daylight spectra. ${ }^{17}$ For surface reflectance we use either the basis functions given by Cohen ${ }^{18}$ for a set of Munsell chips or a set of basis vectors found by our own principal-component analysis for the set of natural reflectances cataloged by Krinov. ${ }^{19}$ For camera filters we use Kodak filters No. 25 (red), No. 58 (green), and No. $47 \mathrm{~B}$ (blue). ${ }^{20}$

\section{VARIATIONAL EQUATIONS}

\section{A. Assumptions}

Consider the scene consisting of two Lambertian surfaces depicted in Fig. 1, where the illumination is composed of two components: diffuse isotropic radiation and a point source (or possibly an extended source) of unknown location. When both components have the same spectral power distribution (cf. Ref. 21), the illumination $E(\lambda)$ can be approximated as a sum, as in Section 2. Because of the nonisotropic illumination component, the incident

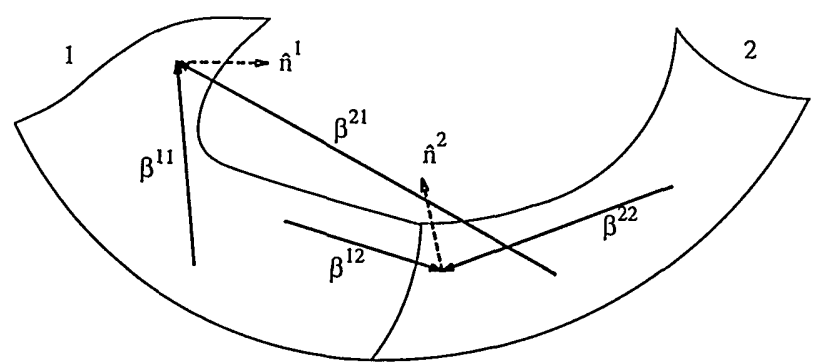

Fig. 1. Each point on a surface has a shading factor that depends on the local normal $\hat{n}$. If only one interreflection bounce is taken into account, a point on surface 1 receives light reflected from all of surface 1 that is proportional to $\beta^{11}$ and from all of surface 2 that is proportional to $\beta^{21}$. 
light intensity varies from point to point by a shading factor resulting from foreshortening.

We lump both the diffuse factor $(\equiv 1)$ and the shading factor for the point source into a single factor $\alpha$. With the surface 2-space parameterized by a set of parameters that we denote simply by $x$, the illumination at a point is

$$
E(x, \lambda)=\alpha(x) E(\lambda)
$$

where we assume that $E(\lambda)$ is constant throughout the scene. It is straightforward to extend the model to include two components of illumination having different spectra, but we shall not do so here. Nevertheless, an extension of the variational formulation introduced below can in fact handle such cases.

Under the one-bounce approximation the interreflection situation in Fig. 1 requires consideration of light reflected from surface 1 to itself and from surface 2 to surface 1 ; the situation is similar for surface 2 . For known surfaces the forward radiosity problem is to calculate the sum of all contributions to mutual illumination at a point $x$. Here we address the inverse problem and seek to derive what these contributions are from the image. Let the factor $\beta^{21}$ comprise all illumination contributions from every point on surface 2 to a small area on surface 1 . Thus $\beta^{21}$ lumps all shape, orientation, and shading factors for mutual illumination into one parameter.

\section{B. One-Bounce Interreflection Equations}

Let us turn now to the general case of many interreflecting surfaces for which the image is of $I=1, \ldots, M$ patches, each of which is assumed to have uniform surface reflectance $S^{I}(\lambda)$. Following Ref. 2, we divide each surface patch into many small subpatches. On the Ith patch (again we denote position simply by $x$ ) the one-bounce approximation to the color signal from the location $x$ is given by

$$
C^{I}(x, \lambda)=\alpha(x) E(\lambda) S^{I}(\lambda)+\sum_{J=1}^{M} \beta^{J I}(x) S^{I}(\lambda) E(\lambda) S^{J}(\lambda) .
$$

In Eq. (5) $\beta^{J I}(x)$ is different for every patch pair. In particular, Eq. (5) includes self-reflection.

We now substitute the finite-dimensional linear model approximations of Section 2 and carry out the integrations of Eq. (4). By straightforward substitution we arrive at camera red-green-blue (RGB) values

$$
\begin{aligned}
\rho_{k}{ }^{I}(x)= & \alpha^{I}(x) \sum_{a} \sum_{\mu} \epsilon_{a} \sigma_{\mu}{ }^{I} g_{a \mu k} \\
& +\sum_{J} \beta^{J I}(x) \sum_{\mu} \sum_{a} \sum_{\nu} \sigma_{\mu}{ }^{I} \epsilon_{a} \sigma_{\nu}{ }^{J} h_{a \mu \nu k},
\end{aligned}
$$

where we denote the spatial parameters for surface $I$ as simply $x$ with patch index $I$ implied $-x$ means $x^{I}$ in a function defined on area $A^{I}$. In practice $x$ is defined on a finite grid in image coordinates and the $\rho_{k}^{I}(x)$ are pixel values at image position $x$ in patch $I$. We assume that scene radiance is proportional to image intensity.

Equation (6) relates the measured sensor responses to the parameters that we wish to recover that describe the surface reflectances and illumination. Let us call the shading $\alpha$ and the interreflection parameters $\beta$ fields, since they take on values throughout regions of the image. The following question arises: How many fields and how many unknown parameters $\epsilon, \sigma$ can we expect to derive from the $\rho$ data available in an image?

For the special case of only two patches with $\beta^{J I}$ that approach zero at some point, we showed ${ }^{10}$ that we could find the fields $\beta^{21}$ and $\beta^{12}$ as well as all the $\epsilon_{\alpha}, \sigma_{\mu}$ up to one overall multiplicative constant, provided that the dimensionalities of the finite-dimensional-model spaces were related by $3 s \geq 2 n+m$.

In solving Eq. (6), the number of unknowns equals the number of values in the $\alpha$ and $\beta$ fields plus the number of $\epsilon$ 's and $\sigma$ 's minus the number of constraints, if any, that can be applied among the fields and minus 1 to account for a single overall multiplicative constant. This constant arises because we cannot derive all values absolutely, since, for example, simultaneously multiplying $\epsilon$ and all the $\beta$ 's by $k$ and dividing all the $\sigma$ 's by $k$ results in the same $\rho$. Balancing these unknowns are the knowns provided by the $s$ components of $\rho$ measured at each $x$ for each of the $M$ patches.

Unfortunately, not all the $\rho$ information may be usable, however. For example, all the $\rho_{k}$ vectors from an isolated patch without interreflection are parallel. If the patch area is $N$ pixels, the number of observations is $s N$, but because of the parallelism these measurements provide only $(s-1)+N$ independent values.

In general, the number of observations per patch varies, but for discussion purposes suppose that we make $N$ measurements of $\rho_{k}$ in every patch. In this case, the total number of measurements is $s M N$. The number of unknowns is given by the $n M$ values of $\sigma$ plus the $m-1$ values of $\epsilon$ (one of the components of $\epsilon$ can be set to 1 to pin down the arbitrary multiplicative constant) plus the $M N F-C$ values from the $F$ fields that we wish to determine less the $C$ constraints obtaining among them. Therefore we must have

$$
n M+(m-1)+M N F-C \leq s M N .
$$

This inequality fixes an upper bound on the number of fields $F$ that can be recovered. As long as $F$ is not chosen too large, the inequality will be satisfied; however, in solving the resulting set of equations, not all the matrices involved may be invertible because of degenerate data. Given a limited number (usually only three) of sensor classes, differences in color signal spectra that in principle could be extracted may collapse into dependent sensor values. Finally, the above inequality condition is certainly necessary for recovery of intrinsic properties of lights and surfaces but is not necessarily sufficient if the set of equations to be solved is nonlinear.

\section{Minimization Integral}

A natural method of solving Eq. (6) for the continuous $\alpha$ and $\beta$ fields is to rewrite it as a minimization problem and apply the calculus of variations. In this case, the quantity to minimize is the integral of the sum of squares of all the deviations of the measured $\rho_{k}$ from the corresponding versions of the right-hand side of Eq. (6).

To simplify the resulting expression we adopt a modified form of the Einstein summation convention: Repeated subscript indices for multiplicative factors imply summa- 
tion, but superscript indices are not summed over unless summation is explicitly shown. We also adopt tensor notation: The quantity $\rho_{k}$ stands for the $k$ th component of the vector $\boldsymbol{\rho}$ as well as the whole collection of components making up the vector.

With these conventions the minimization integral is

$$
\begin{aligned}
\widetilde{\mho} \equiv \sum_{I} \int\left[\rho_{k}{ }^{I}(x)-\alpha^{I}(x) \epsilon_{\alpha} \sigma_{\mu}{ }^{I} g_{a \mu k}\right. \\
\\
\left.\quad-\sum_{J} \beta^{J I}(x) \sigma_{\mu}{ }^{I} \epsilon_{a} \sigma_{\nu}{ }^{I} h_{a \mu \nu k}\right]^{2} \mathrm{~d} A^{I} .
\end{aligned}
$$

The notation [ $]^{2}$ is a shorthand for norm squared in $s$-dimensional space,

$$
\boldsymbol{\rho}^{2} \equiv\left[\rho_{k}\right]^{2} \equiv \sum_{k} \rho_{k} \rho_{k} \equiv \rho_{k} \rho_{k},
$$

where the inner product of two vectors $\rho_{k}$ and $\tau_{k}$ is written as $\rho_{k} \tau_{k}$.

Since $\alpha$ and $\beta$ are unknown functions that minimize the above integral, we can generate equations for them by taking variational derivatives of $\mathfrak{F}$. Solutions for such equations will be given as algebraic functions of $\epsilon$ and $\sigma$.

Let us assume for the present that all the functions $\beta$ are independent. If this assumption is wrong, it may be corrected by the addition of further terms to $\mathfrak{\mho}$. In Section 4 we insert such constraints on the basis of knowledge of the physical meaning of $\beta$ and show how such constraints modify the variational problem.

For each patch $I$ and at each point $x$ in the patch, we set the variational derivative with respect to $\alpha$ equal to zero (see, e.g., Ref. 22):

$$
\begin{array}{r}
\frac{\delta \mathfrak{F}}{\delta \alpha^{I}(x)} \\
=-2\left[\rho_{k}{ }^{I}(x)-\alpha^{I}(x) \epsilon_{a} \sigma_{\mu}{ }^{I} g_{a \mu k}-\sum_{J} \beta^{J I}(x) \sigma_{\mu}{ }^{I} \epsilon_{a} \sigma_{\nu}{ }^{J} h_{a \mu \nu k}\right] \\
\times\left[\epsilon_{b} \sigma_{\omega}{ }^{I} g_{b \omega k}\right]=0 .
\end{array}
$$

Viewing the above Euler-Lagrange equation as giving $\alpha$ in terms of all the other variables, we can immediately find the solution

$$
\alpha^{I}(x)=\frac{\left[\rho_{k}{ }^{I}(x)-\sum_{J} \beta^{J I}(x) \sigma_{\mu}{ }^{I} \epsilon_{\alpha} \sigma_{\nu}{ }^{J} h_{a \mu \nu h}\right]\left[\epsilon_{b} \sigma_{\omega}{ }^{I} g_{b \omega k}\right]}{\left[\epsilon_{b} \sigma_{\omega}{ }^{I} g_{b \omega k}\right]^{2}} .
$$

The assumption built into the variational problem of Eq. (7) is that any observed $\rho$-vector is made up of a term proportional to illumination times reflectance, the primary component of the sensor values, plus terms corresponding to illumination times reflectance times an additional reflectance factor - the one-bounce components. Equation (9) states that the first of these, the no-bounce signal, has a shading factor $\alpha$ found by projecting the portion of $\rho$ not attributable to mutual illumination onto the no-bounce $\rho$-vector directions.

Similar variational derivatives for the $\beta^{J I}$ give

$$
\begin{aligned}
& \frac{\delta \mathfrak{F}}{\alpha \beta^{J I}(x)} \\
& =-2\left[\rho_{k}{ }^{I}(x)-\alpha(x) \epsilon_{a} \sigma_{\mu}{ }^{I} g_{a \mu k}-\sum_{K} \beta^{K I}(x) \sigma_{\mu}{ }^{I} \epsilon_{a} \sigma_{\nu}{ }^{K} h_{a \mu \nu k}\right] \\
& \times\left[\epsilon_{b} \sigma_{\omega}{ }^{I} \sigma_{\tau}{ }^{J} h_{b \omega \tau k}\right]=0 . \quad(10)
\end{aligned}
$$

This is a matrix equation for all the $\beta^{J I}$ for a particular $I$, as can be seen be rewriting it in the form

$$
\begin{aligned}
& {\left[\sum_{K} \beta^{K I}(x) \epsilon_{a} \sigma_{\mu}{ }^{I} \sigma_{\nu}{ }^{K} h_{a \mu \nu k}\right]\left[\epsilon_{b} \sigma_{\omega}{ }^{I} \sigma_{\tau}{ }^{J} h_{b \omega \tau k}\right]} \\
& =\left[\rho_{k}{ }^{I}(x)-\alpha^{I}(x) \epsilon_{a} \sigma_{\mu}{ }^{I} g_{a \mu k}\right]\left[\epsilon_{b} \sigma_{\omega}{ }^{I} \sigma_{\tau}{ }^{J} h_{b \omega \tau k}\right] .
\end{aligned}
$$

The $\beta^{J I}$ are given in terms of projections of those parts of the $\rho_{k}$ not accounted for by the primary color signal direction onto the one-bounce direction.

Taken together, Eqs. (8) and (10) restate the major assumption that vectors $\rho_{k}$ may be composed from a set of linearly independent vectors in the primary color signal $\rho_{k}$ direction and in the set of one-bounce $\rho_{k}$ directions.

For the unknown constants $\epsilon_{a}$ and $\sigma_{\mu}{ }^{I}$, the minimization consists of an ordinary least-squares problem. Accordingly, we take partial derivatives of $\mathfrak{F}$ to obtain normal equations. For the surface reflectance parameters $\sigma$ we find that

$$
\begin{aligned}
\frac{\partial \widetilde{f}}{\partial \sigma_{\mu}{ }^{I}}= & -2 \sum_{J} \int\left\{\left[\rho_{k}{ }^{J}(x)-\alpha^{J}(x) \epsilon_{a} \sigma_{\nu}{ }^{J} g_{a \nu k}\right.\right. \\
& \left.-\sum_{K} \beta^{K J}(x) \epsilon_{a} \sigma_{\nu}{ }^{J} \sigma_{\omega}{ }^{K} h_{a \nu \omega k}\right] \\
& \times\left(\delta^{I J}\left[\alpha^{I} \epsilon_{b} g_{b \mu k}+\sum_{L} \beta^{L I} \epsilon_{b} \sigma_{\tau}{ }^{L} h_{b \mu \tau k}\right]\right. \\
& \left.\left.+\left[\beta^{I J} \epsilon_{b} \sigma_{\tau}{ }^{J} h_{b \mu \tau k}\right]\right)\right\} \mathrm{d} A^{J}=0,
\end{aligned}
$$

where $\delta^{I J}$ is the Kronecker delta and equals 1 for $I=J$ and 0 otherwise. Note that the $I$ th term in the above sum is the most complex and also contributes the most to the sum. Both that term and the other terms contribute to the self-reflection from patch $I$ onto itself. Equations (12) constitute $M$ sets of $n$ coupled equations in $n$ unknowns for each patch.

The similar equations formed by taking partial derivatives with respect to the $\epsilon_{a}$ are

$$
\begin{aligned}
\frac{\partial \mathfrak{F}}{\partial \epsilon_{a}}= & -2 \sum_{I} \int\left\{\left[\rho_{k}{ }^{I}(x)-\alpha^{I}(x) \epsilon_{b} \sigma_{\mu}{ }^{I} g_{b \mu k}\right.\right. \\
& \left.-\sum_{K} \beta^{K I}(x) \epsilon_{b} \sigma_{\mu}{ }^{I} \sigma_{\nu}{ }^{K} h_{b \mu \nu k}\right] \\
& \left.\times\left[\alpha^{I} \sigma_{\omega}{ }^{I} g_{a \omega k}+\sum_{L} \beta^{L I} \sigma_{\omega}{ }^{L} \sigma_{\tau}{ }^{I} h_{a \omega \tau k}\right]\right\} \mathrm{d} A^{I}=0 .
\end{aligned}
$$

\section{Reciprocity Constraints}

By examining the exchange of power between patches, we showed ${ }^{10}$ that in some cases the one-bounce interreflection factor $\beta^{21}$ can be identified with a configuration factor. ${ }^{2}$ A configuration factor is a single-bounce, diffuse-reflector quantity that describes the ratio of the power reaching a small area $\Delta A^{1}$ from another surface $A^{2}$ normalized in terms of the total radiation leaving $A^{2}$. This factor incorporates all geometric factors and is integrated over all of area $A^{2}$. In Ref. 2 the configuration factor is denoted $\mathrm{d} F_{2-d 1}$, meaning that the configuration factor contains a differential and that it sums up the geometrical relationship between a finite area 2 and a differential area 1 . Specifically, we showed that one could make the identification $\mathrm{d} F_{2-d 1}=\Delta A^{1} \beta^{21} / A^{2}$, provided that (a) there is no shading field present and (b) the small element of sur- 
face 1 sees all of surface 2 . From the reciprocity relation for configuration factors, $A^{2} \mathrm{~d} F_{2-d 1}=\Delta A^{1} \mathrm{~d} F_{d 1-2}$, we in fact also can make the identification $\beta^{21}=\mathrm{d} F_{d 1-2}$, where $\mathrm{d} F_{d 1-2}$ gives the ratio of radiation leaving $\Delta A^{1}$ that reaches $A^{2}$ to the total radiation from $\Delta A^{1}$ into the hemisphere above it. Note that this identification holds only for situations without shading and without occluding surfaces blocking surface 2 from surface 1 .

The view factor $v^{21}$ between elements of two surfaces is defined to be unity if the element on surface 1 can see the element on surface 2 and zero otherwise. ${ }^{7}$ By taking view factors and shading into account, we may express the interreflection factor $\beta^{J I}(x)$, which relates all of surface $J$ to a small area on surface $I$, in terms of a configuration factor:

$$
\beta^{J I}(x)=\int_{A^{J}} v^{J I}(x, y) \alpha^{J}(y) \mathrm{d} F_{\mathrm{d} I-\mathrm{d} J},
$$

where $y$ parameterizes surface $J$ and $\mathrm{d} F_{\mathrm{d} I-\mathrm{d} J}$ is a surfaceelement-to-surface-element configuration factor. (Note that the apparent transposition of $I$ and $J$ in $\mathrm{d} F_{\mathrm{d} I-\mathrm{d} J}$ comes about from substitution of a reciprocity condition relating configuration factors and differential areas into the power balance. ${ }^{2}$ )

When the conditions of no shading (i.e., diffuse illumination) and no occlusion (view factor always 1 ) are met, then

$$
\beta^{J I}(x)=\int_{A^{J}} \mathrm{~d} F_{\mathrm{d} I-\mathrm{d} J} \equiv \mathrm{d} F_{\mathrm{d} I-J}
$$

Integration of configuration factors leads to form factors expressing the radiative exchange between finite surfaces: $F_{1-2}=\left(1 / A^{1}\right) \int_{A^{1}} \mathrm{~d} F_{\mathrm{d} 1-2} \mathrm{~d} A^{1}$. A reciprocity relation exists between form-factor pairs as well: $A^{1} F_{1-2}=A^{2} F_{2-1}$. Substituting the identifications of $\beta^{I J}$ leads to a constraint on values of $\beta^{J I}$ and $\beta^{I J}$ as follows:

$$
\int_{A^{I}} \beta^{J I}\left(x^{I}\right) \mathrm{d} A^{I}=\int_{A^{J}} \beta^{I J}\left(x^{J}\right) \mathrm{d} A^{J} .
$$

This constraint can be added to the minimization problem as an integral constraint, with constant Lagrange multiplier $\Lambda$, by

$$
\mathfrak{\mho} \rightarrow \mathfrak{F}-\Lambda\left[\int_{A^{I}} \beta^{J I}\left(x^{I}\right) \mathrm{d} A^{I}-\int_{A^{J}} \beta^{I J}\left(x^{J}\right) \mathrm{d} A^{J}\right] .
$$

Reciprocity relations exist for every pair of patches. However, Eq. (16) does not constrain the self-reflection field $\beta^{I I}$, so in total for $M$ patches there are $1 / 2 M(M-1)$ extra constraints that arise from reciprocity considerations. For these constraints to apply, the correspondence must hold between the one-bounce interreflection factor and the geometric configuration factor, which means that shading and occlusions must not be present. The relationship between $\beta$ and the configuration factor must also hold, but we can expect it to be violated somewhat when there is a large component of light owing to mutual illumination; in that case $\beta$ must account for the significant proportion of the light from multiple bounces within a one-bounce model. In Section 5 we simulate scenes for which the reciprocity constraint can be used, ones with not too substantial a percentage of mutual illumination, only diffuse illumination, and no occlusions.

\section{E. Integrals Replaced by Sums}

The integral formulation of the continuous case provides a unifying framework for finding the determining equations for the minimization. In practice we cannot use Eqs. (12) and (13) as they stand, because when we are presented with an image we do not know the actual values of $\mathrm{d} A^{I}$ in the scene. However, we may simply replace the integrals by sums by dropping all the $\mathrm{d} A^{I}$ and treating all pixels as evenly weighted. The units of area in Eqs. (12) and (13) are unforeshortened area; configuration factors are ratios of image irradiance, i.e., power per unit of area and not per steradian (cf. Ref. 23). Because the surfaces are Lambertian, the appearance of the scene is proportional to irradiance. The integrated interreflection factor in Eq. (15) is the ratio of energy arriving at (unforeshortened) $\mathrm{d} A^{I}$ from all of surface $J$ to all the energy leaving surface $J$. The imaging system responds to radiance, i.e., power per unit of foreshortened area per steradian. If the camera sees an area $\mathrm{d} A$ associated with one pixel and that area corresponds to $f \mathrm{~d} A$ of actual area, then foreshortening simply divides by $f$, and the interreflection factor is unchanged.

\section{TEST ON A SYNTHETIC SCENE}

The equations of Section 3 describe the general case of interreflection among an arbitrary number of surfaces. To test the algorithm developed from those equations, we must specialize to the case of a particular scene. Published analytic solutions for image intensity distributions arising from interreflection are limited to simple configurations of surfaces. Since we need to know all the scene parameters to compare with those recovered by our algorithm, we are constrained to choices of simple configurations of surfaces for our test. Therefore, as a test scene, we consider two convex patches illuminated by diffuse light and write out the above equations for this particular case. For this configuration the fields $\beta^{12}$ and $\beta^{21}$ are related by a reciprocity relation, so it becomes necessary to add an integral constraint term to the minimization integral. In Section 5 we test the equations developed here by specializing further to two planar surfaces because results for that simple configuration are known.

\section{A. Two Convex Patches, Diffuse Illumination}

For the case of two surfaces under diffuse illumination, Eqs. (8)-(13) simplify in several ways. First, the shading field $\alpha$ drops out because there is no shading under isotropic illumination. (Section 5 further specializes to a scene with interreflection between two planes; for planes, testing with additional illumination from a distant point source would not have much advantage over testing with only diffuse illumination because on the planes the shading, and therefore $\alpha$, would be a constant on each surface.) With convex surfaces there will be no self-reflection, so the only remaining one-bounce fields are $\beta^{12}$ and $\beta^{21}$. Under these circumstances Eq. (11) becomes

$$
\beta^{21}(x)=\frac{\left\{\left[\rho_{k}{ }^{1}(x)-\epsilon_{a} \sigma_{\mu}{ }^{1} g_{a \mu k}\right]\left[\epsilon_{b} \sigma_{\nu}{ }^{1} \sigma_{\omega}{ }^{2} h_{b \nu \omega k}\right]\right\}}{\left\{\left[\epsilon_{d} \sigma_{\tau}{ }^{1} \sigma_{\kappa}{ }^{2} h_{d \tau \kappa k}\right]^{2}\right\}},
$$

and there is a similar equation for $\beta^{12}$ with 1 exchanged everywhere with 2 . 
For $\sigma_{\mu}{ }^{1}$, Eqs. (12) reduce to

$$
\begin{aligned}
& \int\left[\rho_{k}{ }^{1}\left(x^{1}\right)-\epsilon_{a} \sigma_{\nu}{ }^{1} g_{a \nu k}-\right.\left.\beta^{21}\left(x^{1}\right) \epsilon_{a} \sigma_{\nu}{ }^{1} \sigma_{\omega}{ }^{2} h_{a \nu \omega k}\right] \\
& \times\left[\epsilon_{b} g_{b \mu k}+\beta^{21}\left(x^{1}\right) \epsilon_{b} \sigma_{\tau}{ }^{2} h_{b \mu \tau k}\right] \mathrm{d} A^{1} \\
&+\int\left[\rho_{k}{ }^{2}\left(x^{2}\right)-\epsilon_{a} \sigma_{\nu}{ }^{2} g_{a \nu k}-\beta^{12}\left(x^{2}\right) \epsilon_{a} \sigma_{\nu}{ }^{1} \sigma_{\omega}{ }^{2} h_{a \nu \omega k}\right] \\
& \times\left[\beta^{12}\left(x^{2}\right) \epsilon_{b} \sigma_{\tau}{ }^{2} h_{b \mu \tau k}\right] \mathrm{d} A^{2}=0
\end{aligned}
$$

where now we show $I=1$ or $I=2$ on the set of parameters $x^{I}$ that parameterize area $A^{I}$. The equations for $\sigma_{\mu}^{2}$ are found by exchanging 1 with 2 in Eq. (19).

The $\epsilon_{a}$ equations, Eqs. (13), become

$$
\begin{aligned}
& \int\left[\rho_{k}{ }^{1}\left(x^{1}\right)-\epsilon_{b} \sigma_{\mu}{ }^{1} g_{b \mu k}-\beta^{21}\left(x^{1}\right) \epsilon_{b} \sigma_{\mu}{ }^{1} \sigma_{\nu}{ }^{2} h_{b \mu \nu k}\right] \\
& \times\left[\sigma_{\omega}{ }^{1} g_{a \omega k}+\beta^{21}\left(x^{1}\right) \sigma_{\omega}{ }^{1} \sigma_{\tau}{ }^{2} h_{a \omega \tau k}\right] \mathrm{d} A^{1} \\
& +\int\left[\rho_{k}{ }^{2}\left(x^{2}\right)-\epsilon_{b} \sigma_{\mu}{ }^{2} g_{b \mu k}-\beta^{12}\left(x^{2}\right) \epsilon_{b} \sigma_{\mu}{ }^{1} \sigma_{\nu}{ }^{2} h_{b \mu \nu k}\right] \\
& \quad \times\left[{\sigma_{\omega}}^{2} g_{a \omega k}+\beta^{12}\left(x^{2}\right) \sigma_{\omega}{ }^{1} \sigma_{\tau}{ }^{2} h_{a \omega \tau k}\right] \mathrm{d} A^{2}=0 .
\end{aligned}
$$

Equation (18) states that once the $\epsilon_{a}$ and $\sigma_{\mu}{ }^{I}$ are fixed, the interreflection factors are given algebraically and follow from the component of the $\rho_{k}$ not explained by the primary $\rho_{k}$ that comes from the color signal.

Equations (19) and (20) are matrix equations: Eq. (19) can be viewed as $n$ equations in the $n$ unknowns $\sigma_{\mu}{ }^{1}$; similarly, Eq. (20) is a set of $m$ equations in the $m$ unknowns $\epsilon_{a}$. In fact, to solve these equations given $\rho_{k}(x)$, we must solve Eqs. (18)-(20) simultaneously for all the unknowns, $\beta^{12}, \beta^{21}, \epsilon_{a}, \sigma_{\mu}{ }^{1}$, and $\sigma_{\mu}{ }^{2}$, such that all the equations are satisfied. In keeping with the discussion of Subsection 3.E, we note that, to convert Eqs. (19) and (20) to summation equations, we simply drop the $d A$ 's and replace integrals by sums.

\section{B. Symmetry Group}

By inspection one sees that the above set of equations remains satisfied if one solution set is transformed into another by the transformation

$$
\begin{aligned}
\epsilon_{a} & \rightarrow Z \epsilon_{a}, \\
\sigma_{\mu}{ }^{1} & \rightarrow \frac{\sigma_{\mu}{ }^{1}}{Z}, \\
\sigma_{\mu}{ }^{2} & \rightarrow \frac{\sigma_{\mu}{ }^{2}}{Z}, \\
\beta^{21}\left(x^{1}\right) & \rightarrow Z \beta^{21}\left(x^{1}\right), \\
\beta^{12}\left(x^{2}\right) & \rightarrow Z \beta^{12}\left(x^{2}\right) .
\end{aligned}
$$

Since the above transformation constitutes a group, one may in principle be able to simplify the set of equations and even to derive solutions by using group theory alone (see Ref. 24 and references cited therein). For our current purposes, it is enough to note that one arbitrary multiplicative constant is available for scaling all the unknowns. We choose the scaling

$$
\epsilon_{1} \equiv 1
$$

\section{Dimensionalities of Illumination and Reflectance Vectors}

There are presumably many different ways to solve the set of nonlinear equations (19) and (20); however, it is not guaranteed that every algorithm will converge. We proceed by dividing the problem into solving each of Eqs. (19) and (20) in turn with each matrix equation treated as a linear, easily solved set of equations in $\sigma$ and $\epsilon$, respectively.

Inspection of Eq. (19) shows that as a matrix equation for $\sigma^{1}$ it can be rewritten as

$$
\sigma_{\nu}{ }^{1} L_{\nu h} M_{k \mu}=N_{\mu}
$$

where $L$ is an $n$ by $s$ matrix, $M$ is an $s$ by $n$ matrix, and $N$ is a 1 by $n$ vector. Thus the matrix that must be inverted to solve for $\sigma^{1}$, viz., $L M$, can have at most rank $s$.

A similar statement can be made for Eq. (20). Since we wish to use an ordinary color camera with $s=3$, in what follows we take

$$
n=m=3 \text {. }
$$

Thus the present method does not permit higher dimensionalities than those arrived at in our previous work. ${ }^{11}$

\section{Reciprocity Constraint}

The reciprocity constraint of Subsection 3.D applies here because of the absence of shading and occlusions. For two surfaces, Eq. (17) takes the form

$$
\mathfrak{\Im} \rightarrow \mathfrak{F}-2 \Lambda\left(\int_{A_{1}} \beta^{21}\left(x^{1}\right) \mathrm{d} A^{1}-\int_{A_{2}} \beta^{12}\left(x^{2}\right) \mathrm{d} A^{2}\right) .
$$

The equations for $\epsilon$ and $\sigma$ are unaffected by this substitution, but Eq. (18) now becomes

$$
\beta^{21}(x)=\frac{\Lambda+\left[\rho_{k}{ }^{1}(x)-\epsilon_{a} \sigma_{\mu}{ }^{1} g_{a \mu k}\right]\left[\epsilon_{b} \sigma_{\nu}{ }^{1} \sigma_{\omega}{ }^{2} h_{b \nu \omega k}\right]}{\left[\epsilon_{d} \sigma_{\tau}{ }^{1} \sigma_{\kappa}{ }^{2} h_{d \tau \kappa k}\right]^{2}} .
$$

The equation for $\beta^{12}$ is similar, but $\Lambda$ is replaced by $-\Lambda$.

Since $\Lambda$ is a constant, we can solve for it. Turning the integral in Eq. (16) into a sum amounts to weighting all pixel data evenly, as was stated in Subsection 3.E. The reciprocity constraint of Eq. (16) becomes

$$
\sum_{A_{1}} \beta^{21}\left(x^{1}\right)=\sum_{A_{2}} \beta^{12}\left(x^{2}\right)
$$

If $N_{1}$ pixels are measured on side 1 and $N_{2}$ pixels are measured on side 2, then substitution of Eq. (18') into Eq. (16') gives the simple expression

$$
\Lambda=\frac{N_{2}\left[\bar{\rho}_{k}{ }^{2}-\epsilon_{a} \sigma_{\mu}{ }^{2} g_{a \mu k}\right]\left[\epsilon_{b} \sigma_{\nu}{ }^{1} \sigma_{\tau}{ }^{2} h_{b \nu \tau k}\right]-N_{1}\left[\bar{\rho}_{k}{ }^{1}-\epsilon_{a} \sigma_{\mu}{ }^{1} g_{a \mu k}\right]\left[\epsilon_{b} \sigma_{\nu}{ }^{1} \sigma_{\tau}{ }^{2} h_{b \nu \tau k}\right]}{N_{1}+N_{2}},
$$


where $\bar{\rho}_{k}{ }^{I}$ is the average of the vector $\rho_{k}{ }^{I}$ over $A^{I}$. This value of $\Lambda$ must be substituted into Eq. (18') whenever the $\beta$ 's are calculated. For more than two patches, similar constraints occur for every pair patch.

\section{E. Algorithm}

To solve the system of nonlinear equations, we apply the same strategy as used in Refs. 25 and 26 by noting that Eq. (19) is linear insofar as the vector $\sigma^{1}$ is concerned. As a result, $\sigma^{1}$ is easily found, provided that all other variables are initialized. Similarly, Eq. (20) is linear in $\epsilon$. Since by Eq. (18') both functions $\beta$ are given algebraically in terms of the measured $\rho$ 's and the variables $\epsilon$ and $\sigma$, an iterative solution is possible.

Our algorithm uses alternating linear equations for $\epsilon$ and the $\sigma$ 's; using the current approximation of the set of numbers $\epsilon$ and $\sigma$, we find a new approximation for the fields $\beta$ from Eq. (18'). Then we iterate and again find a simultaneous solution for $\epsilon$ and $\sigma$ while holding the set of fields $\beta$ fixed. This kind of algorithm is similar to those used in Ref. 27 for solving sets of equations derived from partial differential equations. The scheme is as follows:

\section{Initialize}

1.1. Set $\epsilon=(1,0,0)$.

1.2. Find the best first components of $\sigma^{1}$ and $\sigma^{2}$ by $\sigma_{1}^{I}=\left[\bar{\rho}^{I} \cdot \bar{\rho}^{I}\right] /\left[g_{11} \cdot \bar{\rho}^{I}\right]$, where $\bar{\rho}^{I}$ is the average of $\rho^{I}$ over area $A^{I}$ and $g_{11}$ is the vector with components $g_{11 k}$.

1.3. Find $\beta^{21}$ and $\beta^{12}$ by Eq. (18').

2. While $\epsilon, \boldsymbol{\sigma}^{1}, \boldsymbol{\sigma}^{2}$ and $\beta^{21}, \beta^{12}$ are not converged

2.1. Repeat

2.1.1. Solve Eqs. (19) and (20) in turn for $\epsilon$, $\boldsymbol{\sigma}^{1}, \boldsymbol{\sigma}^{2}$

Until vectors $\epsilon, \boldsymbol{\sigma}^{1}, \boldsymbol{\sigma}^{2}$ are simultaneous solutions for Eqs. (19) and (20) for the current functions $\beta^{21}$ and $\beta^{12}$.

2.2. Find $\beta^{21}, \beta^{12}$ by Eq. (18').

\section{F. Multiplicity of Solutions}

Since the set of equations (19) and (20) is nonlinear, we can expect nonunique solutions. In particular, whereas we are guaranteed that the integral $\mathfrak{\mho}$ is being brought to an extremum, it may in fact be being maximized instead of minimized. Although we have not pursued them, direct methods, such as those in Ref. 28, which produce global minima, might be usefully applied to this problem.

Another possibility is to solve Eqs. (19) and (20) as an algebraic problem. To investigate the complexity of this approach, suppose that we attempt to solve the equations directly by using Cramer's rule. To begin, we substitute for the $\beta^{I J}$ in terms of $\epsilon$ and the $\sigma$ 's, from Eq. (18'). Then, by using the dimensionalities stated above, suppose that we were first to solve Eq. (19) for the $\sigma^{1}$-vector. Then we could substitute the resulting algebraic expression for $\sigma_{\mu}{ }^{1}$ into the equations for $\sigma_{\mu}^{2}$ and solve for the $\sigma^{2}$-vector. In terms of $\sigma^{2}, \beta^{21}$ is a rational function with numerator of degree 1 and denominator of degree 2 , whereas in terms of $\sigma^{1}$ it is a second-degree function over a second-degree function. As well, the solution of Eq. (19) for $\sigma^{1}$ is already a complex rational function of $\sigma^{2}$ even without including the nonlinearities in the $\beta^{I J}$. Therefore the fully simplified version of an equation for component $\sigma_{\mu}{ }^{I}$ will be a polynomial of fairly high degree. Multiple roots are to be expected.

We found that in tests using simulated edges (see Section 5) the system of equations did indeed sometimes proceed to maximize the integral $\mathfrak{\mho}$. When this occurred, we discarded those solutions and changed the initialization. We also found solutions for which the recovered $\epsilon$ was far from the possible vectors associated with illumination vectors. These solutions were also discarded. For each test we found only one solution that was not ruled out by these filtering criteria; the solutions retained were found to produce reasonable agreement with the correct data.

\section{SIMULATIONS}

We tested the model by simulating the edge depicted in Fig. 2, in which two semi-infinite planes of different widths and different surface reflectances meet with opening angle $\gamma$. The field $\beta^{21}$ takes into account all the reflection contributions from area $A^{2}$ to the small area $\Delta A^{1}$. The complete infinite-bounce color signal was calculated by using the full-spectrum radiosity method. Sensor values $\rho_{k}^{I}$ were then calculated by integration with a camera's spectral sensitivity functions. In the resulting image the sensor values vary in a nonlinear fashion across each side and approach no-interreflection values far from the edge. ${ }^{11}$

\section{A. Natural Surfaces}

To test our method we carried out the above algorithm with a synthesized edge having $\gamma=135^{\circ}$ as input and made the width of surface 2 twice that of surface 1 . As a first example, we consider an edge composed of two natural reflectances. In particular, we choose Krinov No. 54 (river valley with meadows) $^{19}$ and Krinov No. 248 (sand dunes) illuminated by standard illumination $\mathrm{A} .{ }^{29}$ In the algorithm's implementation we use the daylight basis of Judd et $a l .{ }^{17}$ and the Munsell chip basis of Cohen ${ }^{18}$ to avoid

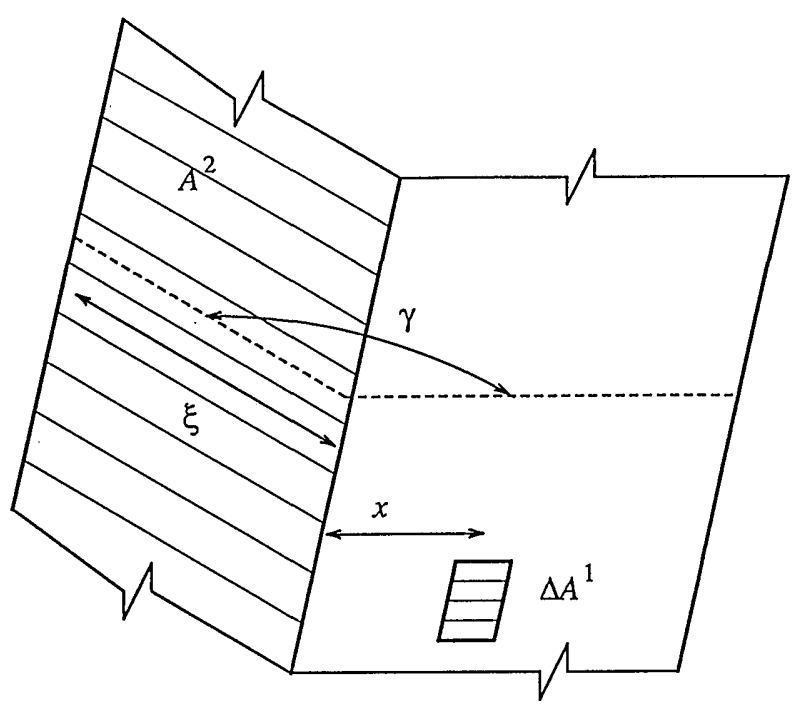

Fig. 2. For planar surfaces under diffuse illumination there is no shading factor or self-reflection. The amount of light reflected from all of a semi-infinite surface $A^{2}$ onto a small area $\Delta A^{1}$ of a semi-infinite surface $A^{1}$ depends only on the distance $x$ of the spot $\Delta A^{1}$ from the edge compared with the width $\xi$ of $A^{2}$. 
Table 1. Errors in Recovered Spectra from a Two-Plane Edge

\begin{tabular}{|c|c|c|c|c|c|c|c|}
\hline & & \multicolumn{2}{|c|}{ Algorithm } & \multicolumn{2}{|c|}{ Algorithm Scaled } & \multicolumn{2}{|c|}{ Best-Fit Model } \\
\hline & & $\mathscr{E}(\%)$ & $\Delta E$ & $\mathscr{E}(\%)$ & $\Delta E$ & $\mathscr{E}(\%)$ & $\Delta E$ \\
\hline \multirow[t]{4}{*}{ (a) } & \multicolumn{7}{|c|}{ Two natural reflectances and Cohen basis } \\
\hline & Illumination & 6.12 & 2.87 & 5.93 & 2.87 & 5.68 & 4.06 \\
\hline & Reflectance 1 & 8.46 & 1.28 & 8.45 & 1.28 & 8.11 & 2.92 \\
\hline & Reflectance 2 & 2.98 & 1.03 & 2.56 & 1.08 & 2.39 & 1.11 \\
\hline \multirow[t]{4}{*}{ (b) } & \multicolumn{7}{|c|}{ Two natural reflectances and Krinov principal-component basis } \\
\hline & Illumination & 5.98 & 3.42 & 5.87 & 3.42 & 5.68 & 4.06 \\
\hline & Reflectance 1 & 7.69 & 1.49 & 7.68 & 1.48 & 6.99 & 2.95 \\
\hline & Reflectance 2 & 5.05 & 1.94 & 4.91 & 2.00 & 3.87 & 3.47 \\
\hline \multirow[t]{4}{*}{ (c) } & \multicolumn{7}{|c|}{ Pantone paper and Munsell principal-component basis } \\
\hline & Illumination & 5.81 & 3.99 & 5.78 & 3.99 & 5.68 & 4.06 \\
\hline & Pink & 10.77 & 6.19 & 10.75 & 6.14 & 10.56 & 5.12 \\
\hline & Green & 23.25 & 16.45 & 23.21 & 16.34 & 21.08 & 8.18 \\
\hline \multirow[t]{9}{*}{ (d) } & \multicolumn{7}{|c|}{$\begin{array}{l}\text { Two natural reflectances with opening angle of } 45^{\circ} \text { and Cohen basis } \\
\text { Illumination }\end{array}$} \\
\hline & No noise & 5.97 & 3.14 & 5.85 & 3.14 & 5.68 & 4.06 \\
\hline & With noise & 6.06 & 2.74 & 5.89 & 2.74 & & \\
\hline & Reflectance 1 & & & & & & \\
\hline & No noise & 18.03 & 3.99 & 8.90 & 0.68 & 8.11 & 3.58 \\
\hline & With noise & 17.97 & 4.00 & 8.93 & 0.65 & & \\
\hline & Reflectance 2 & & & & & & \\
\hline & No noise & 7.86 & 2.57 & 2.72 & 1.26 & 2.39 & 1.05 \\
\hline & With noise & 7.77 & 2.55 & 2.75 & 1.21 & & \\
\hline
\end{tabular}

any direct relationship between the spectra in the synthesized scene and the finite-dimensional models approximating them.

As an error measure for judging the accuracy of the recovered spectra, we use the percentage error defined as $\mathscr{E}=\left\{\Sigma[f(\lambda)-\tilde{f}(\lambda)]^{2} / \Sigma[f(\lambda)]^{2}\right\}^{1 / 2}$, where $\tilde{f}(\lambda)$ is a function that approximates $f(\lambda){ }^{25}$ We also use the CIELUV ${ }^{29}$ colordifference formula $\Delta E$. Here we use a three-dimensional $\Delta E$ that measures changes in intensity as well as in chromaticity.

Section (a) of Table 1 tabulates the errors in the spectra recovered by the algorithm from the synthetic test scene. For columns 2 and 3 the recovered reflectances are unscaled and the recovered illumination is corrected only for the fact that $\epsilon_{1}=1$ was an arbitrary choice. The recovered spectrum is corrected by the $\epsilon_{1}$ weight obtained by a least-squares fit of the illumination basis vectors to the spectrum of standard illuminant A. For the next two columns the recovered spectra have been scaled to minimize the error (see Ref. 25). This factors out intensity, so these columns measure the errors in the relative spectral shapes and as such are a good test of the color constancy obtained. For comparison, the last two columns show the errors for direct least-squares fits of the spectra by the finite-dimensional model. It can be seen that the algorithm does not increase the error much over that created by the finite-dimensional approximation. Figure 3 plots the actual and recovered spectra.

Figure 4 compares the recovered values $\beta$ with what should be expected theoretically. The recovered $\beta$ is scaled by $\epsilon_{1}$ and plotted against a theoretical function $\zeta$ representing the effect of one-bounce interreflection. ${ }^{11}$ When the distance variables $x$ and $\xi$ shown in Fig. 2 are used, $\zeta$ is given by $\zeta=1 / 2\left[1+(\cos \gamma-X) /\left(X^{2}+1-\right.\right.$ $\left.2 X \cos \gamma)^{1 / 2}\right]$, where $X$ is the dimensionless distance
$X=x / \xi$. We can expect $\beta$ to resemble this $\zeta$; however, the recovered $\beta$ is offset from this $\zeta$.

Because $\beta$ encompasses multiple interreflections within a one-bounce model, whereas $\zeta$ is calculated for the theoretical case of exactly one bounce, we should usually expect $\beta$ to be greater than $\zeta$ (cf. Ref. 11). However, the offset in recovered $\beta$ values is from another source: inaccuracy in recovering the no-interreflection color signal for each side. In Eq. (6) we have modeled $\rho_{k}$ on each side of the edge as sweeping out a plane between two vectors: (a) the vector in RGB color space that would be observed from that surface in the absence of interreflection and (b) the RGB vector corresponding to interreflection alone. The planes for both sides intersect in the latter $\rho_{k}$ vector. ${ }^{12}$ If the algorithm produces a small error in the estimate of the no-interreflection vector, then the error vector, projected into the plane, results in an extra contribution in the interreflection color direction that accounts for an additive error in the prediction of $\beta$ values.

To quantify how closely the recovered $\beta$ matches the theoretical one-bounce function $\zeta$, we can use an error measure similar to the measure $\mathscr{E}$ that we used for spectra (although we do not expect $\beta$ to match $\zeta$ exactly). We find $\mathscr{E}=1.35 \%$ on side 1 of the edge and $\mathscr{E}=2.61 \%$ on side 2 for $\beta$ shifted by a constant offset. (These values can be reduced by offsetting and scaling as well as by $\beta \rightarrow A \beta+B$; then $\mathscr{E}$ becomes $0.4 \%$ on both surfaces.) If we alter the algorithm by requiring $\beta$ to go to zero far from the edge, the interreflection factors recovered are almost exactly equal to these offset functions. In that case the illumination and reflectance spectra recovered are little changed from those recovered without applying such boundary conditions to the $\beta$ 's.

Changing the basis sets that we use for reflectance and illumination should lead to somewhat different results. 


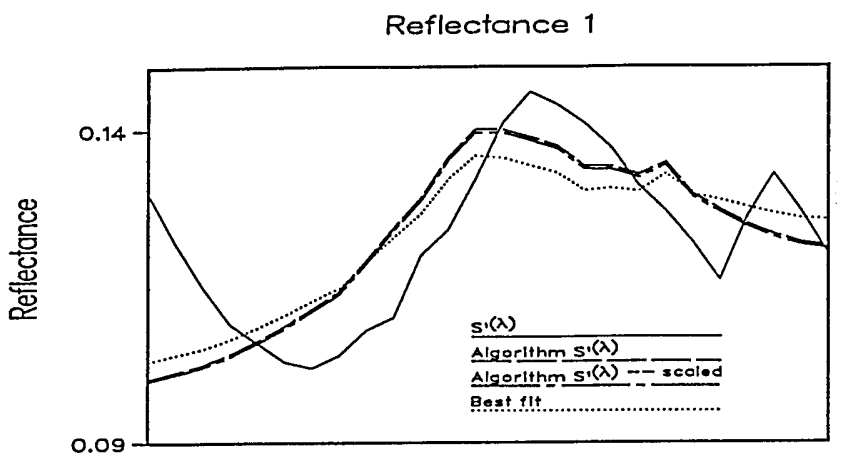

Reflectance 2

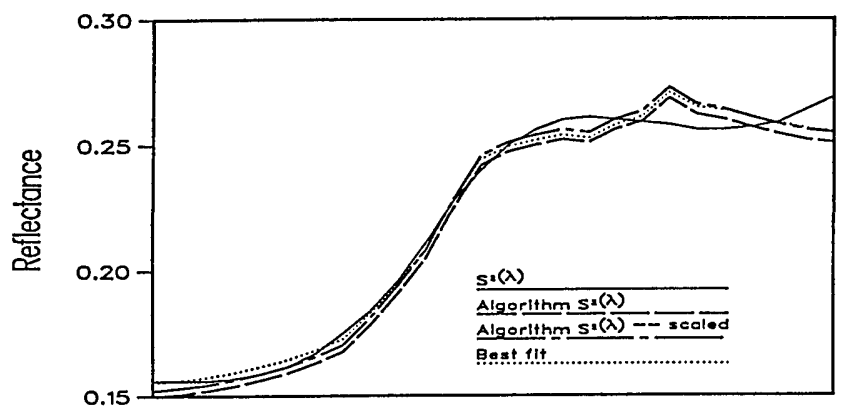

llumination

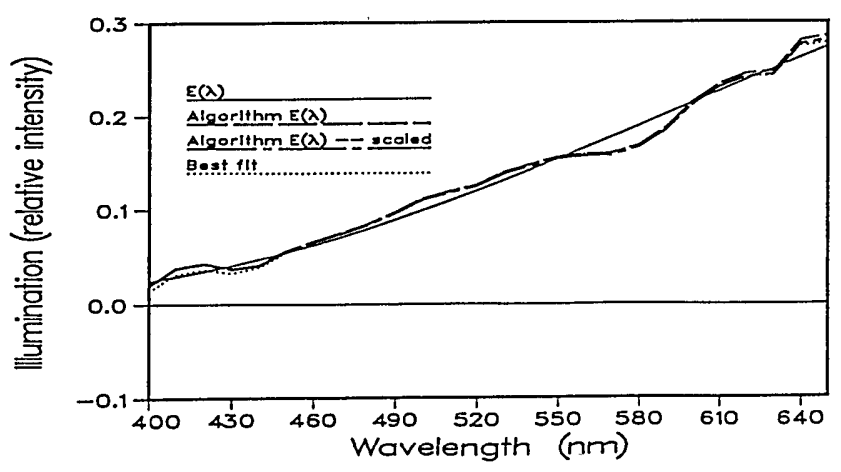

Fig. 3. Recovered spectra for illumination and the two surface reflectances for the two-plane edge of Fig. 2. Error data are given in section (a) of Table 1. Shown are the actual spectrum (-), the recovered spectrum including brightness information (--), the recovered spectrum scaled to give the best match to the actual spectrum (---), and the best fit available within a finitedimensional model of dimension $3(\cdots)$.

In particular, if we use a reflectance basis set that better models the reflectances we actually expect to see in an image, then we might expect to do better.

Section (b) of Table 1 gives the results for the same reflectances as above but uses reflectance basis vectors derived from a principal-component analysis that we carried out on a set of more than $300 \mathrm{Krinov}$ spectra (see Ref. 15). One might expect that, when the reflectances found in the scene are drawn from the same set as was used in the principal-component analysis, the algorithm might work better; however, whereas the basis vectors explain the variance for a large set of data, they do not necessarily work well for any particular reflectance function. We see from section (b) of Table 1 that, whereas reflectance 1 is modeled slightly better by the Krinov basis, reflectance 2 is modeled slightly worse. In the end the algorithm recovers the illumination spectrum with approximately the same accuracy as before. For the reflec- tance spectra the algorithm does better when the finite-dimensional model does also. Note that the error measure $\Delta E$ is nonlinear and does not strictly follow improvements in the error $\mathscr{E}$.

For the functions $\beta$ we find error values $\mathscr{E}=0.82 \%$ and $\mathscr{E}=2.01 \%$ on sides 1 and 2 , respectively. For scaled curves these figures become $0.44 \%$ and $0.36 \%$.

\section{B. Man-Made Surfaces}

As another test the spectral reflectance functions of Pantone color-standard paper were measured. Section (c) of Table 1 shows the results of applying our algorithm to the two-plane edge of Fig. 2 composed of pale pink and emerald green paper (Pantone No. 217 and No. 334) illuminated by standard illuminant A. We used the Munsellchip basis for this test and found that, even though the green paper is poorly modeled by it, the algorithm still does reasonably well in recovering the spectra that are better modeled. For the $\beta$ 's the errors for sides 1 and 2 were $\mathscr{E}=7.29 \%$ and $\mathscr{E}=1.57 \%$, respectively. These errors become $1.9 \%$ and $0.4 \%$ for the scaled curves.

\section{Noise}

To study the behavior of the algorithm with respect to noise, we repeated the simulation of section (a) of Table 1 with the edge opening angle reduced to $45^{\circ}$ and with both reflectances normalized to equal brightnesses. These changes increase the amount of interreflection between the two planes such that it becomes responsible for $10 \%$ to $24 \%$ of the image intensity on the short-plane side of the edge and for $1 \%$ to $23 \%$ on the long-plane side.

To add noise we first shift and scale each channel of the whole image into the range 0 to 255 . A uniform distribu-

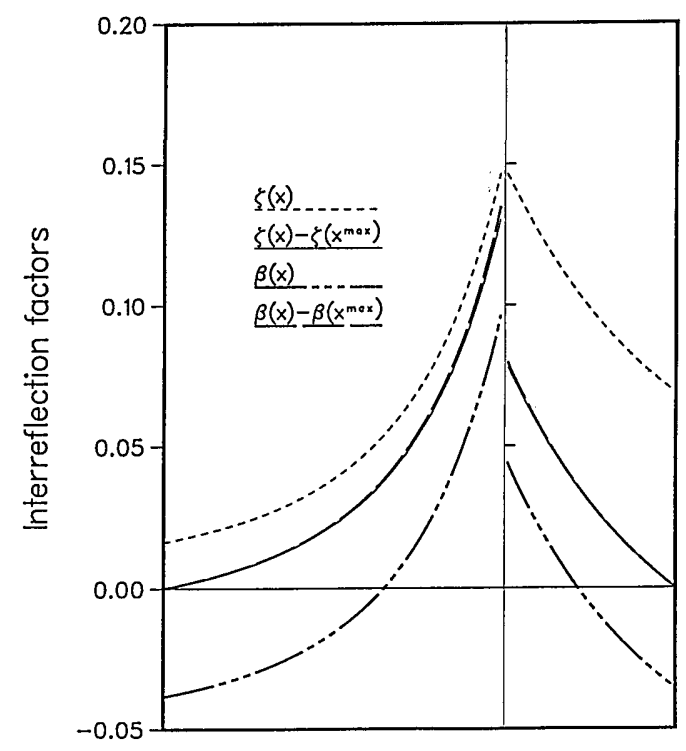

\section{Surface 2}

Distance from edge

\section{Surface 1}

Fig. 4. Interreflection factors recovered by the algorithm for the two-plane edge of Fig. 2 with the width of surface 2 equal to twice the width of surface 1 . The algorithm recovers the function $\beta$. On surface 1 this is $\beta^{21}$, and on surface 2 it is $\beta^{12}$. For comparison we show the factor $\zeta$ derived from the theory for a single bounce. The curves for $\beta$ and $\zeta$ agree well once $i$ inth curves are offset such that they both go to zero at the pixel farthest from the edge. 


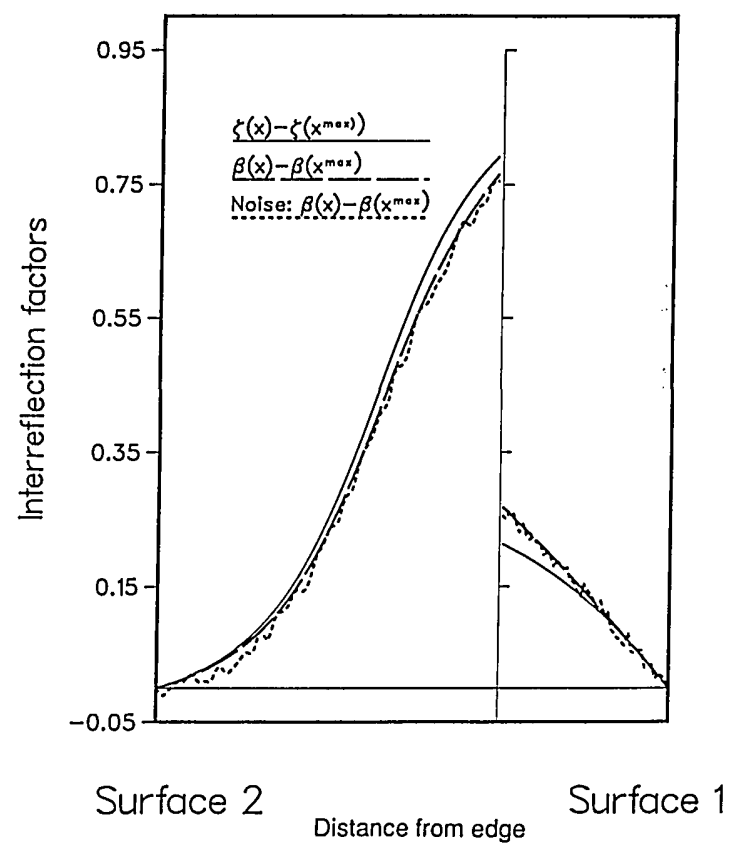

Fig. 5. Interreflection factors for the two-plane edge with opening angle $45^{\circ}$ : theoretical one-bounce configuration factor $\zeta(-)$, recovered interreflection factor $\beta(--), \beta$ recovered from noisy data $(\cdots)$.

tion of additive noise is then added in the range \pm 5 out of 256. This noise is then mapped back into the original unshifted data; the result is noise equal to between $2 \%$ and $63 \%$ of the interreflection effect itself. The larger values come about because this level of noise is comparable with the interreflection effect far from the edge. At the edge, where interreflection is strongest, noise amounts to $2-4 \%$ of the RGB contributions from interreflection. The results of running the algorithm on input RGB values with noise added are shown in section (d) of Table 1 . The results are essentially unaffected by the addition of noise.

Figure 5 shows the behavior of the interreflection factor $\beta$ in the presence of noise. Rather than comparing the results for $\beta$ with the theoretical factor $\zeta$, in this case we compare the results for $\beta$ with noise with these same results without noise. Again we make use of $\mathscr{E}$ as a convenient measure of the percentage deviation of one curve from another. The measure of the residuals $\&$ for $\beta$ with noise compared with those without noise is $5.17 \%$ on side 1 and $3.42 \%$ on side 2 .

\section{CONCLUSIONS}

In an image the effects of the direct illumination, interreflected illumination, and surface reflectance intermix in a complex manner. As a result, a general theoretical analysis of image intensities in terms of these components is bound to be complicated.

In a simple gray-level image too much information is collapsed into a single intensity value for the interreflection effects to be calculated; however, in a three-band color image, color shifts created by interreflection allow the analysis to proceed. By applying the calculus of variations, finite-dimensional models of illumination and surface reflectance, and a one-bounce model of interreflection, we derived equations that can be solved for the scene's illumi- nation, surface reflectances, interreflection fields, and shading fields. The theory describes the general case of many different interreflecting surfaces under the assumptions that the image is segmented into regions of equal reflectance, all reflectances are Lambertian, and there is only one illuminant spectrum.

Testing the theory presents some difficulties in that a meaningful test requires that all the parameters of the scene be known. In particular, to apply the algorithm to the image of a real scene is easy, but to know that the results are correct requires a fully calibrated camera system and a fully calibrated scene in the sense that the complete spectrum of the illuminant and the percentspectral-reflectance functions of all surfaces must be known. We do not yet have means for acquiring the necessary data. In addition, a calibrated scene still does not provide any direct interreflection-factor or shading-factor information.

Conducting tests on a synthetic image also poses problems in that it requires a method of synthesizing an image for specified scene parameters. We chose a scene for which a published solution for the image-intensity distribution exists, ${ }^{11}$ with interreflection factors that are close to one-bounce configuration factors. For this scene the general-case equations specialized to simpler ones, which were then encoded as an algorithm. The algorithm's performance was good even when random noise was added.

The algorithm is useful in that it extracts several intrinsic scene properties. Interesting, but perhaps least important, is the spectrum of the illumination. The recovered surface reflectances, however, are important because they are independent of the illumination and therefore provide a solution for color constancy when there is measurable interreflection. The recovered interreflection field represents the component of the image intensity created by interreflection, and as such it can be removed from the image to create a shading field that is the same as it would have been if there had been no interreflection. This corrected shading can then be used for shape from shading.

\section{ACKNOWLEDGMENTS}

This research was supported by grants from the Centre for Systems Science of Simon Fraser University and the Natural Sciences and Engineering Research Council of Canada.

\section{REFERENCES}

1. J. E. Kaufman, IES Lighting Handbook, 4th ed. (Illuminating Engineering Society, New York, 1966).

2. R. Siegel and J. R. Howell, Thermal Radiation Heat Transfer (Hemisphere, New York, 1981).

3. E. M. Sparrow and R. D. Cess, Thermal Radiation Heat Transfer (Hemisphere, New York, 1978).

4. J. J. Koenderink and A. J. van Doorn, "Geometrical modes as a general method to treat diffuse interreflections in radiometry," J. Opt. Soc. Am. 73, 843-850 (1983).

5. D. Forsyth and A. Zisserman, "Mutual illumination," in Proceedings: Computer Vision and Pattern Recognition (Institute of Electrical and Electronics Engineers, New York, 1989), pp. 466-473.

6. D. Forsyth and A. Zisserman, "Shape from shading in the light of mutual illumination," Image Vis. Comput. 8, 42-49 (1990). 
7. S. K. Nayar, K. Ikeuchi, and T. Kanade, "Shape from interreflections," in Proceedings: International Conference on Computer Vision (Institute of Electrical and Electronics Engineers, New York, 1990), pp. 2-11.

8. C. M. Goral, K. E. Torrance, D.P. Greenberg, and B. Battaile, "Modeling the interaction of light between diffuse surfaces," Comput. Graph. 18, 213-222 (1984).

9. R. Hall, Illumination and Color in Computer Generated Imagery (Springer-Verlag, New York, 1989).

10. B. V. Funt, M. S. Drew, and J. Ho, "Color constancy from mutual reflection," Int. J. Comput. Vis. 6, 5-24 (1991).

11. M. S. Drew and B. V. Funt, "Calculating surface reflectance using a single-bounce model of mutual reflection," in Proceedings: International Conference on Computer Vision (Institute of Electrical and Electronics Engineers, New York, 1990), pp. 393-399.

12. B. V. Funt and M. S. Drew, "Color space analysis of mutual illumination," IEEE Trans. Patt. Anal. Mach. Intell. (to be published)

13. B. K. P. Horn and M. J. Brooks, "The variational approach to shape from shading," Computer Vis. Graph. Image Process. 33, 174-208 (1986).

14. L. T. Maloney and B. A. Wandell, "Color constancy: a method for recovering surface spectral reflectance," J. Opt. Soc. Am. A 3, 29-33 (1986).

15. L. T. Maloney, "Evaluation of linear models of surface spectral reflectance with small numbers of parameters," J. Opt. Soc. Am. A 3, 1673-1683 (1986).

16. B. A. Wandell, "The synthesis and analysis of color images," IEEE Trans. Patt. Anal. Mach. Intell. PAMI-9, 2-13 (1987).

17. D. B. Judd, D. L. MacAdam, and G. Wyszecki, "Spectral distribution of typical daylight as a function of correlated color temperature," J. Opt. Soc. Am. 54, 1031-1040 (1964).
18. J. Cohen, "Dependency of the spectral reflectance curves of the Munsell color chips," Psychon. Sci. 1, 369-370 (1964).

19. E. L. Krinov, Spectral Reflectance Properties of Natural Formations, Technical Translation TT-439 (National Research Council of Canada, Ottawa, 1947).

20. Kodak Filters for Scientific and Technical Uses, 2nd ed. (Eastman Kodak Company, Rochester, N.Y., 1981).

21. R. Gershon, A. D. Jepson, and J. K. Tsotsos, "Ambient illumination and the determination of material changes," J. Opt. Soc. Am. A 3, 1700-1707 (1986).

22. R. Courant and D. Hilbert, Methods of Mathematical Physics (Interscience, New York, 1962).

23. B. K. P. Horn, Robot Vision (MIT, Cambridge, Mass., 1986).

24. M. S. Drew, S. Kloster, and J. D. Gegenberg, "Lie group analysis and similarity solutions for the generalized Liouville equation," Nonlinear Anal. 13, 489-505 (1989).

25. J. Ho, B. V. Funt, and M. S. Drew, "Separating a color signal into illumination and surface reflectance components: theory and applications," IEEE Trans. Patt. Anal. Mach. Intell. 12, 966-977 (1990).

26. B. V. Funt, J. Ho, and M. S. Drew, "Method and apparatus for determining ambient light and surface reflectance," U.S. patent 4,992,963 (February 12, 1991).

27. J. R. Rice and R. F. Boisvert, Solving Elliptic Problems Using ELLPACK (Springer-Verlag, Berlin, 1985).

28. A. A. Amini, T. E. Weymouth, and R. C. Jain, "Using dynamic programming for solving variational problems in vision," IEEE Trans. Patt. Anal. Mach. Intell. 12, 855-867 (1990).

29. G. Wyszecki and W. S. Stiles, Color Science: Concepts and Methods, Quantitative Data and Formulas, 2nd ed. (Wiley, New York, 1982). 